\title{
Pien Tze Huang inhibits tumor cell proliferation and promotes apoptosis via suppressing the STAT3 pathway in a colorectal cancer mouse model
}

\author{
QUNCHUAN ZHUANG $^{1,2}$, FEI HONG $^{3}$, ALING SHEN ${ }^{1}$, LIANGPU ZHENG $^{1,2}$, JIANWEI ZENG $^{1,2}$, \\ WEI LIN ${ }^{1,2}$, YOUQIN CHEN ${ }^{4,5}$, THOMAS J. SFERRA ${ }^{4,5}$, ZHENFENG HONG ${ }^{1}$ and JUN PENG ${ }^{1,3}$ \\ ${ }^{1}$ Academy of Integrative Medicine Biomedical Research Center and ${ }^{2}$ Fujian Key Laboratory of Integrative Medicine \\ on Geriatrics, Fujian University of Traditional Chinese Medicine, 1 Huatuo Road, Minhou Shangjie, Fuzhou, Fujian 350108; \\ ${ }^{3}$ Postdoctor Workstation, Zhangzhou Pien Tze Huang Pharmaceutical Co., Ltd., Shangjie, Zhangzhou, Fujian 363000, \\ P.R. China; Departments of ${ }^{4}$ Pediatrics and ${ }^{5}$ Biochemistry and Molecular Biology, The University of Oklahoma \\ Health Sciences Center, 940 Stanton L. Young Boulevard, Oklahoma City, OK 73190, USA
}

Received November 1, 2011; Accepted December 22, 2011

DOI: $10.3892 /$ ijo.2012.1326

\begin{abstract}
Signal transducer and activator of transcription 3 (STAT3) plays a critical role in cell survival and proliferation. Constitutive activation of STAT3 is strongly correlated with pathogenesis of various types of malignant tumors including colorectal cancer (CRC), and therefore is a major focus in the development of anti-cancer agents. Pien Tze Huang (PZH), a well-known traditional Chinese formula prescribed already in the Ming Dynasty, has been demonstrated to be clinically effective in the treatment of CRC. However, the precise mechanism of its anti-cancer activity remains largely unknown. In the present study we evaluated the efficacy of PZH against tumor growth in vivo in the CRC mouse xenograft model, and investigated the underlying molecular mechanisms. We found that administration of PZH reduced tumor volume and tumor weight but had no effect on body weight gain in CRC mice, demonstrating that $\mathrm{PZH}$ can inhibit colon cancer growth in vivo without apparent adverse effect. We also observed that $\mathrm{PZH}$ treatment inhibited the phosphorylation level of STAT3 in tumor tissues. Consequently, the inhibitory effect of PZH on STAT3 activation resulted in the up-regulation of $\mathrm{Bax} / \mathrm{Bcl}-2$ ratio as well as down-regulation
\end{abstract}

Correspondence to: Dr Jun Peng, Academy of Integrative Medicine Biomedical Research Center, 1 Huatuo Road, Minhou Shangjie, Fuzhou, Fujian 350108, P.R. China

E-mail: pjunlab@hotmail.com

Abbreviations: CRC, colorectal cancer; PZH, Pien Tze Huang; STAT3, signal transducer and activator of transcription 3; TCM, traditional Chinese medicine; TUNEL, terminal deoxynucleotidyl transferase-mediated dUTP nick end labeling; PCNA, proliferating cell nuclear antigen

Key words: Pien Tze Huang, Chinese medicine, colorectal cancer, STAT3 pathway, apoptosis, proliferation of Cyclin D1 and CDK4 expression, leading to the induction of apoptosis as well as the inhibition of cell proliferation. These results suggest that promotion of cancer cell apoptosis and inhibition of proliferation via suppression of STAT3 pathway might be one of the mechanisms by which PZH treats colorectal cancer.

\section{Introduction}

Colorectal carcinoma (CRC) is one of the most common human malignancies worldwide, with over 1.2 million new cases and 0.6 million deaths in 2008 (1). To date, chemoprevention is a major therapy for patients with advanced CRC, and 5-fluorouracil (5-FU)-based regimens have been performed as international standard chemotherapy for these patients (2). However, due to drug resistance, patient response to 5-FU-based regimens is less than $40 \%(3,4)$. In addition, many currently used anti-cancer agents will generate unacceptable level of toxicity to normal cells and tissue (4,5). Drug resistance and toxicity limit the effectiveness of current CRC chemotherapy, increasing the necessity for the development of new therapeutic approaches. Natural products, including traditional Chinese medicine (TCM), have received recent interest as therapeutic agents for CRC as they have relatively few side effects and have long been used as alternative remedies for a variety of diseases including cancer (6-14). TCM formula is a complex combination of many natural products, each of which contains numerous chemical compounds. Therefore, TCM formulas are considered to be multi-component and multi-target agents exerting their therapeutic function in a more holistic way; and discovering naturally-occurring agents is a promising approach for anti-cancer treatment.

Signal transducer and activator of transcription 3 (STAT3) is an important transcription factor that plays an essential role in cell survival and proliferation $(15,16)$. STAT3 activation is mediated by phosphorylation at tyrosine 705 by receptor and non-receptor protein tyrosine kinases, such as epidermal growth factor receptor (EGFR) kinase (17) and Janus-activated kinases (JAKs) $(18,19)$. Phosphorylated STAT3 proteins in the cytoplasm dimerize and translocate to the nucleus where they regulate the 
expression of various critical genes involved in cell proliferation and survival (20-22). Constitutive activation of STAT3, resulting in unregulated increase in cell proliferation and reduction in cell apoptosis, is strongly correlated with the development of numerous types of cancer including CRC and commonly suggests poor prognosis (23-28). Therefore, inhibiting cell proliferation and/or promoting apoptosis by suppression of STAT3 activation should be a promising approach in the development of anti-cancer therapies.

Pien Tze Huang (PZH) is a well-known traditional Chinese formulation that was first prescribed by a royal physician in the Ming Dynasty. The main ingredients of PZH include Moschus, Calculus Bovis, Snake Gall, and Radix Notoginseng. These products together confer PZH properties of heat-clearing, detoxification, blood circulation-promotion, blood stasis- and swelling-reduction (29). Since according to the theory of TCM, accumulation of toxic dampness and heat is one of the major causative factors in the pathogenesis of cancers and therefore clearing heat and detoxification is a principle of anti-cancer treatment, $\mathrm{PZH}$ is believed to be an effective anti-cancer agent. In fact, $\mathrm{PZH}$ has been used in China for centuries as a folk remedy for the treatment of various cancers. Modern pharmacological studies proposed that PZH displays therapeutic effects on hepato-cellular carcinoma and colon cancer in clinical trials $(30,31)$; and inhibits the growth of human colon carcinoma cells via activation of mitochondrion-dependent apoptosis (32). However, the precise mechanism of its anti-cancer activity remains largely unclear. To further elucidate its anti-tumor mechanism of action, here we evaluated the efficacy of $\mathrm{PZH}$ against tumor growth in vivo in the CRC mouse xenograft model; and investigated the underlying molecular mechanisms.

\section{Materials and methods}

Materials and reagents. Pien Tze Huang (PZH) was obtained from and authenticated by the sole manufacturer Zhangzhou Pien Tze Huang Pharmaceutical Co. Ltd., China (Chinese FDA approval no: Z35020242). Dulbecco's modified Eagle's medium (DMEM), fetal bovine serum (FBS), penicillin-streptomycin, trypsin-EDTA, TRIzol reagent, were purchased from Invitrogen (Grand Island, NY, USA). SuperScript II reverse transcriptase was provided by Promega (Madison, WI, USA). All the other chemicals, unless otherwise stated, were obtained from Sigma Chemicals (St. Louis, MO, USA).

Preparations of PZH for animal study. Pien Tze Huang (PZH) was obtained from and authenticated by the sole manufacturer Zhangzhou Pien Tze Huang Pharmaceutical Co. Ltd. Just before use, $234 \mathrm{mg}$ of $\mathrm{PZH}$ powder was dissolved in $1 \mathrm{ml}$ saline and then was sonicated for $30 \mathrm{~min}$. The sample was ready for intragastric infusion when the powder was completely dissolved and the solution became clear.

HPLC analysis. PZH powder (300 mg) were extracted with $25 \mathrm{ml}$ of $100 \%$ ethanol for 30 min using refluxing method and filtered. The resultant sample was analyzed on an Agilent 1100 HPLC system (Santa Clara, CA, USA) using a C-18 column. The absorbance was measured at $203 \mathrm{~nm}$ (Fig. 1). The mobile phase consisted of linear gradient $20-40 \%$ acetonitrile in aqueous
$0.5 \%$ phosphoric acid at a flow rate of $1 \mathrm{ml} / \mathrm{min}$ with an injection volume of $10 \mu \mathrm{l}$. A sample containing ginsenoside Rg1, sodium taurocholate and ginsenoside Rb1 was used as control.

Cell culture. Human colon carcinoma HT-29 cells were obtained from American Type Culture Collection (ATCC, Manassas, VA, USA). Cells were grown in DMEM containing $10 \%(\mathrm{v} / \mathrm{v})$ FBS, $100 \mathrm{U} / \mathrm{ml}$ penicillin and $100 \mu \mathrm{g} / \mathrm{ml}$ streptomycin in a $37^{\circ} \mathrm{C}$ humidified incubator with $5 \% \mathrm{CO}_{2}$. The cells were subcultured at $80-90 \%$ confluency.

Animals. Athymic male nude mice were obtained from Vital River Laboratory Animal Technology Co., Ltd. (Beijing, China) and housed in clean pathogen-free rooms in an environment with controlled temperature $\left(22^{\circ} \mathrm{C}\right)$, humidity, and a 12-h light/ dark cycle. Food and water were given ad libitum throughout the experiment. All animal treatments were strictly in accordance with international ethical guidelines and the National Institutes of Health Guide concerning the Care and Use of Laboratory Animals, and the experiments were approved by the Institutional Animal Care and Use Committee of Fujian University of Traditional Chinese Medicine.

In vivo nude mouse xenograft study. HT-29 cells were grown in culture and then detached by trypsinization, washed, and resuspended in serum-free DMEM. Cells $\left(1.5 \times 10^{6}\right)$ mixed with Matrigel (1:1) were subcutaneously injected in the right flank area of athymic nude mice to initiate tumor growth. After 3 days of xenograft implantation, mice were randomized into two groups $(n=12)$ and given intra-gastric administration of $234 \mathrm{mg} /$ $\mathrm{kg} / \mathrm{d}$ dose of PZH or saline daily, 5 days a week for 16 days. Body weight and tumor growth were measured every two days. Tumor growth was determined by measuring the major (L) and minor (W) diameter with a caliper. The tumor volume was calculated according to the following formula: tumor volume $=\pi / 6 \times \mathrm{L} \mathrm{x} \mathrm{W}^{2}$. At the end of experiment, the animals were anaesthetized with pelltobarbitalum natricum, and the tumor issue was removed and weighed.

Immunohistochemstry analysis. Tumor samples were fixed with $10 \%$ formaldehyde for $12 \mathrm{~h}$, and subsequently processed conventionally for paraffin-embedded tumor slides. The slides were subjected to antigen retrieval and the endogenous peroxidase activity was blocked with $3 \%$ hydrogen peroxide in water. For immunohistochemical staining, slides were incubated with rabbit polyclonal antibodies against pSTAT3, PCNA, Bax, Bcl-2, Cyclin D1 or CDK4 (all in 1:200 dilution, Santa Cruz Biotechnology). After washing with PBS, slides were incubated with biotinylated secondary antibody followed by conjugated horseradish peroxidase (HRP)-labelled streptavidin (Dako), and then washed with PBS. The slides were then incubated with diaminobenzidine (DAB, Sigma) as the chromogen, followed by counterstaining with diluted Harris hematoxylin (Sigma). After staining, five high-power fields (400x) were randomly selected in each slide, and the average proportion of positive cells in each field were counted using the true color multi-functional cell image analysis management system (Image-Pro Plus, Media Cybernetics, USA). To rule out any non-specific staining, PBS was used to replace the primary antibody as a negative control. 
A

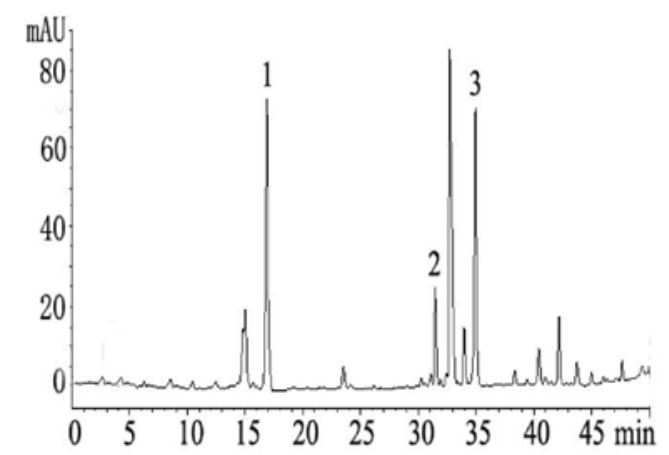

B

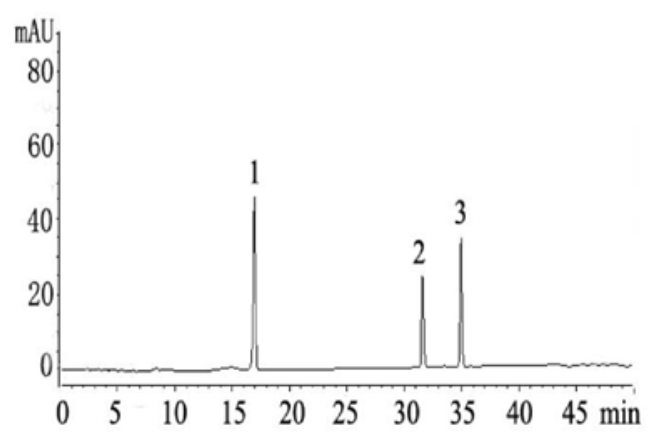

Figure 1. HPLC profiles of PZH (A) and a standard solution (B). The mobile phase consisted of linear gradient $20-40 \%$ acetonitrile in aqueous $0.5 \%$ phosphoric acid. The standard solution was composed of ginsenoside $\operatorname{Rg} 1$ (peak 1), sodium taurocholate (peak 2) and ginsenoside Rb1 (peak 3).

In situ apoptosis detection by terminal deoxynucleotidyl transferase-mediated dUTP nick end labeling (TUNEL) staining. The 5- $\mu \mathrm{m}$-thick sections of tumor samples were analyzed by TUNEL staining using TumorTACS In situ Apoptosis kit (R\&D Systems). Apoptotic cells were counted as DAB-positive cells (brown stained) at five arbitrarily selected microscopic fields at a magnification $\mathrm{x} 400$.

$R T$-PCR. Total RNA from tumor samples was isolated with TRIzol reagent (Invitrogen). Oligo(dT)-primed RNA $(1 \mu \mathrm{g})$ was reverse-transcribed with SuperScript II reverse transcriptase (Promega) according to the manufacturer's instructions. The obtained cDNA was used to determine the mRNA amount of Cyclin D1, CDK4, Bcl-2 and Bax by PCR with Taq DNA polymerase (Fermentas). GAPDH was used as an internal control.

Statistical analysis. All data are the means of three determinations and data were analyzed using the SPSS package for Windows (Version 11.5). Statistical analysis of the data was performed with Student's t-test and ANOVA. Differences with $\mathrm{P}<0.05$ were considered statistically significant.

\section{Results}

PZH inhibits tumor growth in colorectal cancer (CRC) xenograft mice. The in vivo anti-tumor effect of $\mathrm{PZH}$ was evaluated by measuring tumor weight and volume in CRC xenograft mice, while its adverse effect was determined by measuring the body weight gain. As shown in Fig. 2A, PZH treatment resulted in $57 \%$ decrease in tumor volume compared with control $\left(880 \pm 100\right.$ or $375 \pm 100 \mathrm{~mm}^{3}$ per mouse in control or $\mathrm{PZH}$-treated
A

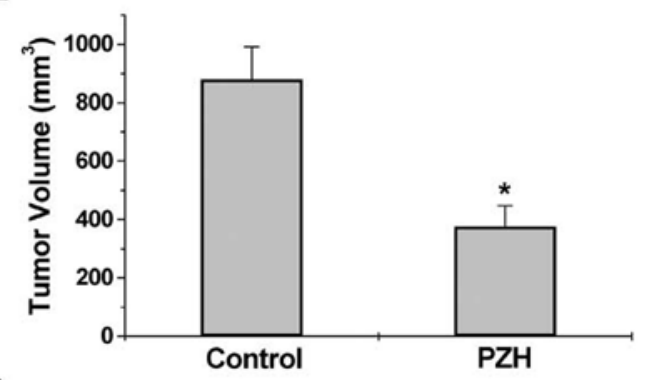

B

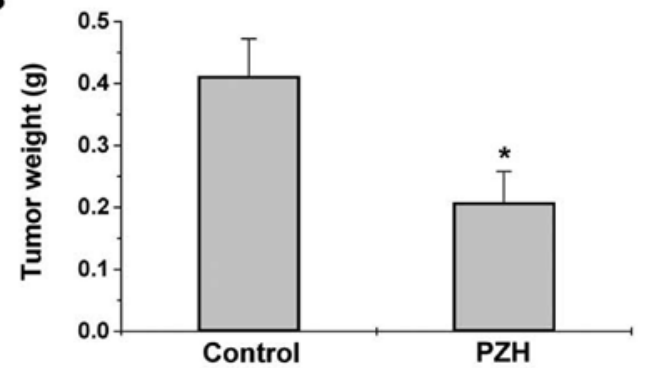

C

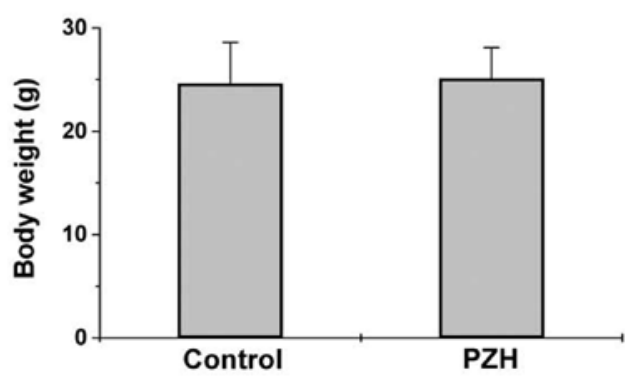

Figure 2. Effect of PZH on tumor growth in mouse xenografts with HT-29 colon cancer cells. After tumor development, the mice were given intra-gastric administration of $234 \mathrm{mg} / \mathrm{kg} / \mathrm{d}$ dose of PZH or PBS daily, 5 days a week for 16 days. Tumor volume (A), tumor weight (B) and body weight (C) were measured at the end of experiment. Data shown are averages with SD (error bars) from 12 mice in each group. ${ }^{*} \mathrm{P}<0.01$, versus controls.

group). Consistently, the tumor weight per mouse in control or PZH-treated group was $0.412 \pm 0.018$ or $0.208 \pm 0.08 \mathrm{~g}$, respectively, accounting for $50 \%$ decrease (Fig. 2B). However, administration of PZH had no effect on the body weight gain in experimental animals (Fig. 2C). Taken together, it is suggested that $\mathrm{PZH}$ is potent in suppressing colon tumor growth in vivo, without apparent signs of toxicity.

PZH inhibits cancer cell proliferation and induces apoptosis in CRC xenograft mice. To determine whether the inhibitory effect of PZH on cancer growth is due to cell proliferation and/or apoptosis, we examined the $\mathrm{PZH}$ pro-apoptotic and anti-proliferative activities in CRC mice via immunohistochemical (IHC) staining for TUNEL and PCNA. Data in Fig. 3 show $24.5 \pm 3.89$ and $41.6 \pm 6.19 \%$ TUNEL-positive cells in control and PZH-treated mouse groups; and the percentage of PCNA-positive cells in control or PZH-treated mice was $34.8 \pm 5.08$ and $23.5 \pm 5.09 \%$, respectively. These data demonstrate that PZH inhibits the proliferation of colon cancer cells and promotes cell apoptosis in vivo.

PZH suppresses STAT3 phosphorylation in CRC xenograft mice. STAT3 plays an important role in cell survival and prolif- 

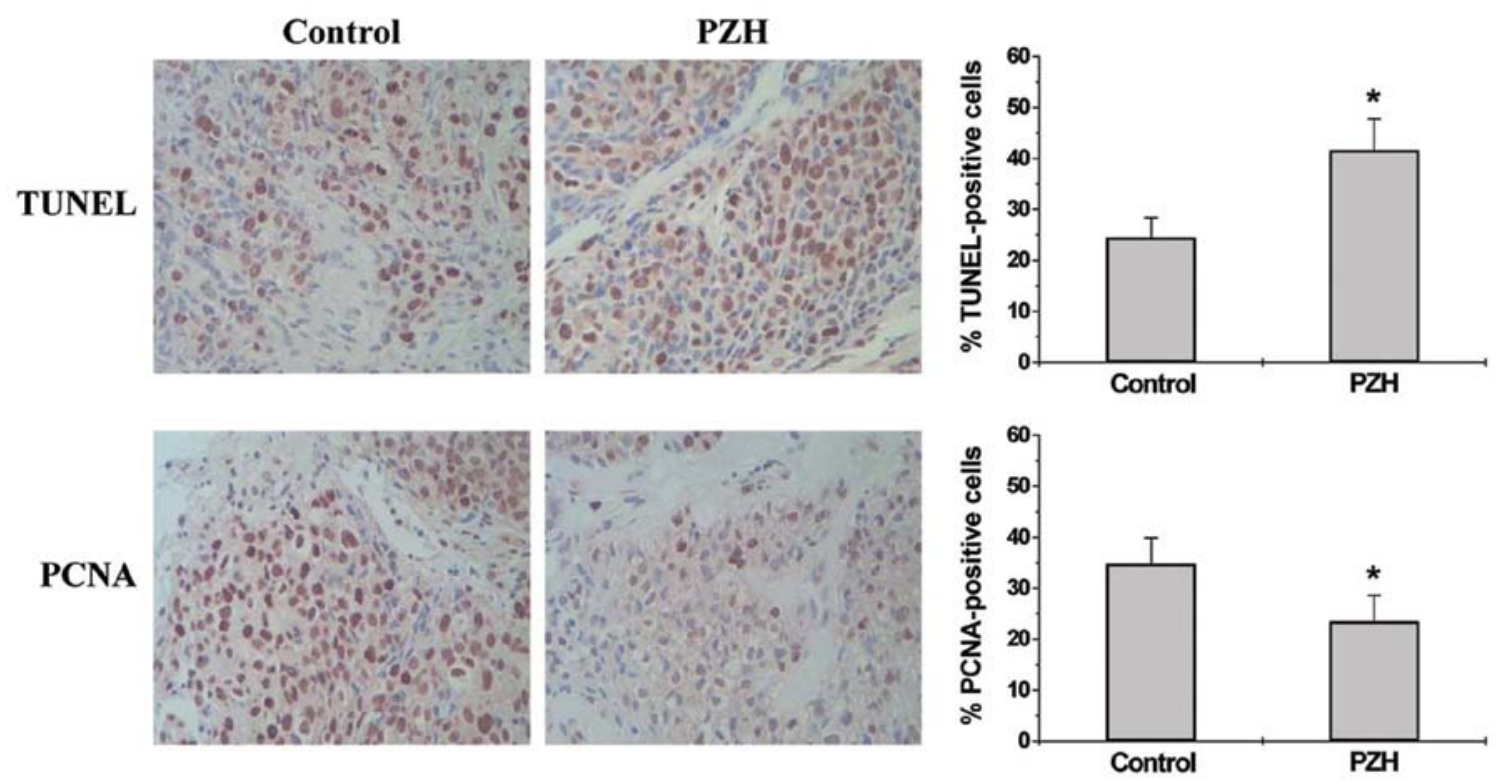

Figure 3. Effect of PZH on cell apoptosis and proliferation in CRC mice. At the end of the experiment, tumor tissues from control and PZH-treated group were processed for immunohistochemical (IHC) staining for TUNEL or PCNA. Representative images were taken at a magnification x400. Quantification of IHC assay is presented as percentage of positively-stained cells. Data shown are averages with SD (error bars) from 12 mice in each group. "P<0.01, versus controls.
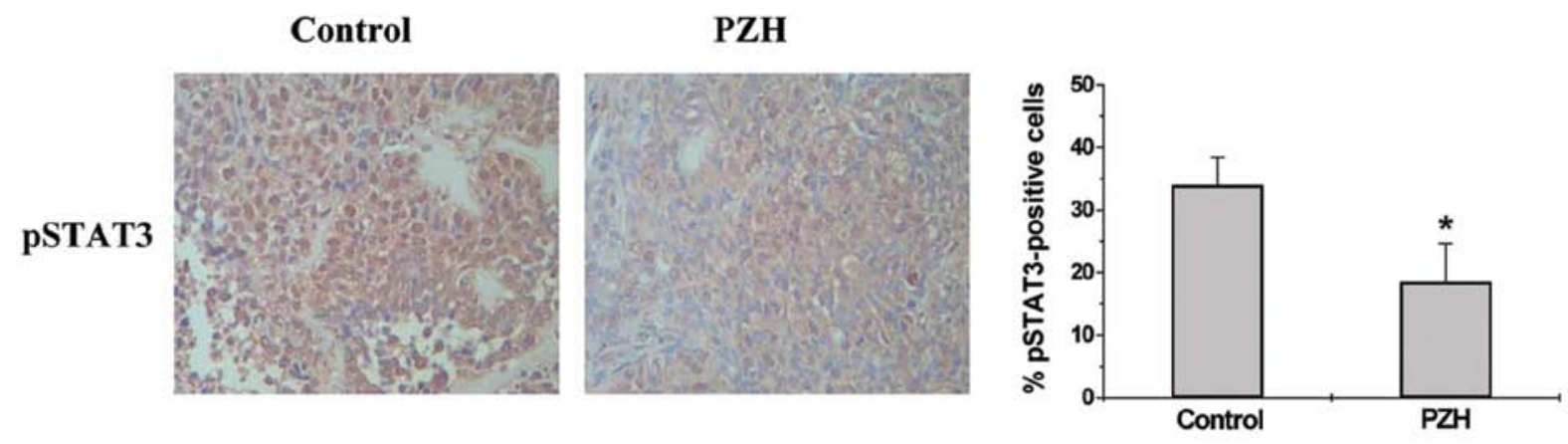

Figure 4. Effect of PZH on phosphorylation of STAT3 in CRC mice. At the end of the experiment, tumor tissues from control and PZH-treated group were processed for IHC assay for phosphorylated STAT3 (pSTAT3). Representative images were taken at a magnification x400. Quantification of IHC assay is presented as percentage of positively-stained cells. Data shown are averages with SD (error bars) from 12 mice in each group. "P<0.01, versus controls.

eration. The phosphorylation/activation of STAT3 (pSTAT3) regulates the expression, leading to promotion of cell proliferation or inhibition of apoptosis. We therefore examined the effect of PZH on STAT3 phosphoralytion in tumor tissue using IHC assay. As shown in Fig. 4, the percentage of pSTAT3positive cells in control or PZH-treated mice was $34.0 \pm 4.38$ and $18.5 \pm 6.21 \%$, respectively $(\mathrm{P}<0.01)$, suggesting that $\mathrm{PZH}$ treatment significantly suppresses the activation of STAT3 in CRC mice.

$P Z H$ regulates the expression of $B c l-2, B a x, C y c l i n D 1$ and $C D K 4$ in CRC xenograft mice. To further explore the mechanism of the PZH pro-apoptotic and anti-proliferative activities, we performed RT-PCR and IHC analyses to, respectively, examine the mRNA and protein expression of Bcl-2, Bax, Cyclin D1 and CDK4 in CRC mice. Results of the RT-PCR showed that PZH treatment reduced the mRNA expression of pro-proliferative
Cyclin D1, CDK4 and anti-apoptotic Bcl-2 in tumors, whereas that of pro-apoptotic Bax was increased (Fig. 5A). Data from IHC assay indicated that the protein expression patterns of Cyclin D1,CDK4, Bcl-2 and Bax were similar to their respective mRNA levels. The percentage of Cyclin D1-, CDK4-, Bcl-2- or Bax-positive cells in control group was $27.5 \pm 3.75,31.8 \pm 5.97$, $25.7 \pm 2.73$ or $20.5 \pm 4.22 \%$, whereas that in PZH-treated mice was $13.8 \pm 5.45,14.3 \pm 5.79,10.8 \pm 5.42$ or $32.3 \pm 6.15 \%$ (Fig. $5 \mathrm{~B}$ ).

\section{Discussion}

Despite advances in the treatment of colorectal cancer (CRC), the overall patient response to chemotherapy is less than $40 \%$ due to drug resistance $(3,4)$. In addition, many currently used anti-cancer agents confer potent intrinsic cytotoxicity to normal cells. Resistance to chemotherapy and cytotoxicity to normal cells limit the effectiveness of current cancer therapies, thus there 
is a need for the development of novel therapeutic agents. Natural products, including traditional Chinese medicine (TCM), have received recent interest as anti-cancer agents since they have relatively few side effects and have been used as alternative remedies for a variety of diseases including cancer. Pien Tze Huang (PZH), a well-known and important TCM formula, has

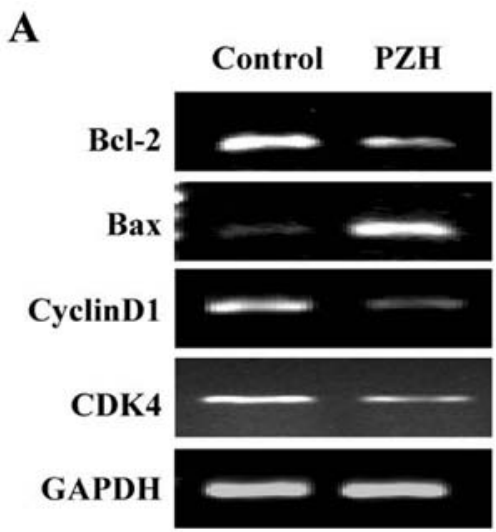

been demonstrated to be clinically effective in treating various cancers including CRC. However, the precise mechanism of its anti-cancer activity remains largely unknown.

The transcription factor STAT3 is essential for cell survival and proliferation. Constitutive activation of STAT3 is one of the major oncogenic pathways involved in the development of CRC and thus becomes a promising therapeutic target (23-28). Using colorectal cancer (CRC) mouse model, here we demonstrated that $\mathrm{PZH}$ could inhibit cancer growth in vivo, without apparent toxicity. In addition, we observed that $\mathrm{PZH}$ suppressed the phosphorylation of STAT3 in tumor tissues. Persistent STAT3 causes unregulated increase in cell proliferation and reduction in apoptosis, leading to development of CRC as well as other types of cancer. Our data in this study showed that suppression of STAT3 activation by PZH treatment resulted in the inhibition of cancer cell proliferation and the promotion of apoptosis in CRC mouse tumor tissues. The proliferation of most animal cells is primarily regulated in the G1/S transition, one of the two main checkpoints in cell cycle which is highly mediated by pro-proliferative Cyclin D1 and CDK4 $(33,34)$. Apoptosis

B
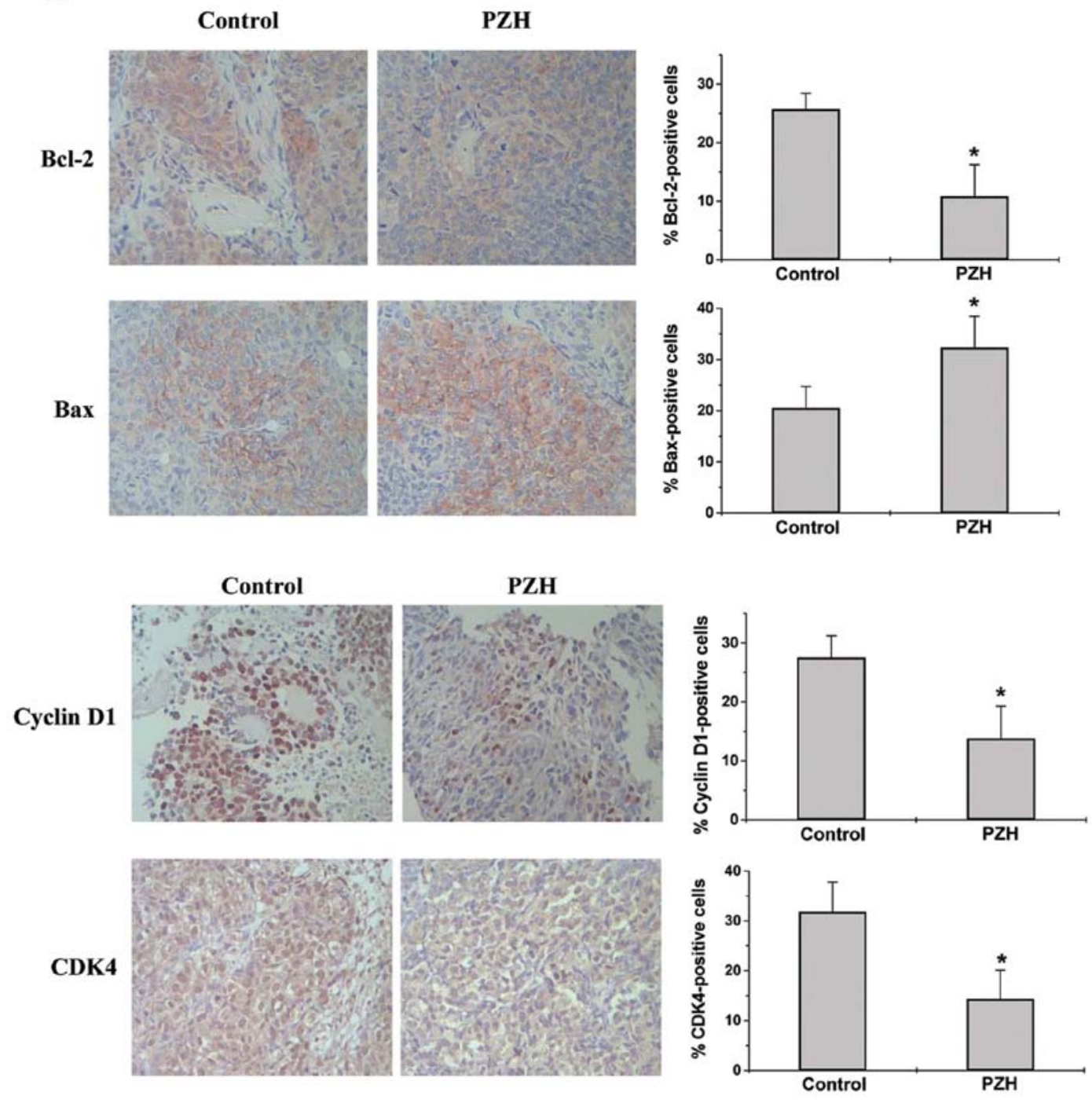

Figure 5. Effect of PZH on the expression of Bcl-2, Bax, Cyclin D1 and CDK4 in CRC mice. (A) The mRNA expression of Bcl-2, Bax, Cyclin D1 and CDK4 in tumor tissues from control and PZH-treated groups was determined by RT-PCR. GAPDH was used as an internal control. (B) The protein expression was analyzed via IHC assay. Representative images were taken at a magnification of x400. Quantification of IHC assay is presented as percentage of positively-stained cells. Data shown are averages with SD (error bars) from 12 mice in each group. ${ }^{*} \mathrm{P}<0.01$, versus controls (B). 
is tightly regulated by Bcl-2 family proteins, including both anti-apoptotic members such as Bcl-2 and pro-apoptotic members such as Bax $(35,36)$. Activated STAT3 participates in the pathogenesis of cancer via modulating the expression of these critical genes involved in cell proliferation and survival, e.g., up-regulating the expression of Cyclin D1 and CDK4, and decreasing the ratio of Bax to Bcl-2 (20-22). Consistent with the inhibitory effect of PZH on STAT3 phosphorylation, our data indicated that administration of $\mathrm{PZH}$ increased the pro-apoptotic Bax/Bcl-2 ratio and inhibited the expression of Cyclin D1 and CDK4 in tumor tissues of CRC mice. In conclusion, for the first time we report that $\mathrm{PZH}$ inhibits colorectal cancer growth in vivo via promotion of cancer cell apoptosis and inhibition of proliferation, which is mediated by the suppression of STAT3 pathway.

\section{Acknowledgements}

This work was sponsored by the National Natural Science Foundation of China (81073097), the Developmental Fund of Chen Keji Integrative Medicine (CKJ 2011001), and the Natural Science Foundation of Fujian Province of China (2010J01195).

\section{References}

1. Jemal A, Bray F, Center MM, Ferlay J, Ward E and Forman D: Global cancer statistics. CA Cancer J Clin 61: 69-90, 2011.

2. Gustin DM and Brenner DE: Chemoprevention of colon cancer: current status and future prospects. Cancer Metast Rev 21: 323-348, 2002.

3. Gorlick R and Bertino JR: Drug resistance in colon cancer. Semin Oncol 26: 606-611, 1999.

4. Longley DB, Allen WL and Johnston PG: Drug resistance, predictive markers and pharmacogenomics in colorectal cancer. Biochim Biophys Acta 1766: 184-196, 2006.

5. Boose G and Stopper H: Genotoxicity of several clinically used topoisomerase II inhibitors. Toxicol Lett 116: 7-16, 2000.

6. Gordaliza M: Natural products as leads to anticancer drugs. Clin Transl Oncol 9: 767-776, 2007.

7. Ji HF, Li XJ and Zhang HY: Natural products and drug discovery. EMBO Rep 10: 194-200, 2009.

8. Zhao J, Jiang P and Zhang WD: Molecular networks for the study of TCM pharmacology. Brief Bioinform 11: 417-430, 2009.

9. Lin JM, Chen YQ, Wei LH, Chen XZ, Xu W, Hong ZF, Sferra TJ and Peng J: Hedyotis Diffusa Willd extract induces apoptosis via activation of the mitochondrion-dependent pathway in human colon carcinoma cells. Int J Oncol 37: 1331-1338, 2010.

10. Peng J, Chen YQ, Lin JM, Zhuang QC, Xu W, Hong ZF and Sferra TJ: Patrinia Scabiosaefolia extract suppresses proliferation and promotes apoptosis by inhibiting STAT3 pathway in human multiple myeloma cells. Mol Med Rep 4: 313-318, 2011.

11. Lin JM, Wei LH, Xu W, Hong ZF, Liu XX and Peng J: Hedyotis Diffusa Willd inhibits angiogenesis in vivo and in vitro: a possible mechanism of its anti-cancer activity. Mol Med Rep 4: 1283-1288, 2011.

12. Wei LH, Chen YQ, Lin JM, Zhao JY, Chen XZ, Xu W, Liu XX, Sferra TJ and Peng J: Scutellaria Barbata D. Don induces apoptosis of human colon carcinoma cell via activation of the mitochondriondependent pathway. J Med Plants Res 5: 1962-1970, 2011.

13. Wei LH, Lin JM, Xu W, Hong ZF, Liu XX, Sferra TJ and Peng J: Inhibition of tumor angiogenesis by Scutellaria barbata D. Don via suppressing proliferation, migration and tube formation of endothelial cells and downregulation of the expression of VEGF-A in cancer cells. J Med Plants Res 5: 3260-3268, 2011.

14. Zheng LP, Chen YQ, Lin W, Zhuang QC, Chen XZ, Xu W, Liu XX, Peng J and Sferra TJ: Spica Prunellae extract promotes mitochondrion-dependent apoptosis in a human colon carcinoma cell line. Afr J Pharm Pharmacol 5: 327-335, 2011.
15. Bromberg $\mathbf{J}$ and Darnell JE Jr: The role of STATs in transcriptional control and their impact on cellular function. Oncogene 19: 2468-2473, 2000.

16. Aggarwal BB, Kunnumakkara AB, Harikumar KB, Gupta SR, Tharakan ST, Koca C, Dey S and Sung B: Signal transducer and activator of transcription-3, inflammation, and cancer: how intimate is the relationship? Ann NY Acad Sci 1171: 59-76, 2009.

17. Zhong Z, Wen Z and Darnell J: Stat3: a STAT family member activated by tyrosine phosphorylation in response to epidermal growth factor and interleukin-6. Science 264: 95-98, 1994.

18. Lutticken C, Wegenka UM, Yuan J, Buschmann J, Schindler C, Ziemiecki A, Harpur AG, Wilks AF, Yasukawa K, Taga T, et al: Association of transcription factor APRF and protein kinase Jak1 with the interleukin-6 signal transducer gp130. Science 263: 89-92, 1994.

19. Migone TS, Lin JX, Cereseto A, Mulloy JC, O'Shea JJ, Franchini G and Leonard WJ: Constitutively activated Jak-STAT pathway in T cells transformed with HTLV-I. Science 269: 79-81, 1995.

20. Darnell JE Jr: STATs and gene regulation. Science 277: 1630-1635, 1997.

21. Zushi S, Shinomura Y, Kiyohara T, Miyazaki Y, Kondo S, Sugimachi M, Higashimoto Y, Kanayama S and Matsuzawa Y: STAT3 mediates the survival signal in oncogenic ras-transfected intestinal epithelial cells. Int J Cancer 78: 326-330, 1998.

22. Masuda M, Suzui M, Yasumatu R, Nakashima T, Kuratomi Y, Azuma K, Tomita K, Komiyama S and Weinstein IB: Constitutive activation of signal transducers and activators of transcription 3 correlates with cyclin D1 overexpression and may provide a novel prognostic marker in head and neck squamous cell carcinoma. Cancer Res 62: 3351-3355, 2002.

23. Bromberg $\mathbf{J}$ and Wang TC: Inflammation and cancer: IL-6 and STAT3 complete the link. Cancer Cell 15: 79-80, 2009.

24. Darnell JE Jr: Transcription factors as targets for cancer therapy. Nat Rev Cancer 2: 740 -749, 2002.

25. Alvarez JV, Greulich H, Sellers WR, Meyerson M and Frank DA: Signal transducer and activator of transcription 3 is required for the oncogenic effects of non-small-cell lung cancer-associated mutations of the epidermal growth factor receptor. Cancer Res 66: 3162-3168, 2006

26. Kusaba T, Nakayama T, Yamazumi K, Yakata Y, Yoshizaki A, Inoue K, Nagayasu T and Sekine I: Activation of STAT3 is a marker of poor prognosis in human colorectal cancer. Oncol Rep 15: 1445-1451, 2006.

27. Lin Q, Lai R, Chirieac LR, Li C, Thomazy VA, Grammatikakis I, Rassidakis GZ, Zhang W, Fujio Y, Kunisada K, Hamilton SR and Amin HM: Constitutive activation of JAK3/STAT3 in colon carcinoma tumors and cell lines: inhibition of JAK3/STAT3 signaling induces apoptosis and cell cycle arrest of colon carcinoma cells. Am J Pathol 167: 969-980, 2005.

28. Xiong H, Zhang Z, Tian X, Sun D, Liang Q, Zhang Y, Lu R, Chen $Y$ and Fang J: Inhibition of JAK1, 2/STAT3 signaling induces apoptosis, cell cycle arrest, and reduces tumor cell invasion in colorectal cancer cells. Neoplasia 10: 287-297, 2008.

29. Chinese Pharmacopoeia Commission: Pharmacopoeia of the Peoples Republic of China. Chinese Medical Science and Technology Press 1: 573-575, 2010.

30. Xu YY and Yu EX: Clinical analysis of the effect of Pien Tze Huang in treatment of 42 patients with moderate or advanced liver cancer. Shanghai J Tradit Chin Med 12: 4-5, 1994.

31. Gu ZX: Therapeutical observation of advanced colon cancer. Chin Tradit Patent Med 15: 23, 1993.

32. Lin JM, Wei LH, Chen YQ, Liu XX, Hong ZF, Sferra TJ and Peng J: Pien Tze Huang-induced apoptosis in human colon cancer HT-29 cells is associated with regulation of the Bcl-2 family and activation of caspase 3. Chin J Integr Med 17: 685-690, 2011.

33. Elledge SJ: Cell cycle checkpoints: preventing an identity crisis. Science 274: 1664-1672, 1996.

34. Taulés M, Rius E, Talaya D, López-Girona A, Bachs O and Agell N: Calmodulin is essential for cyclin-dependent kinase 4 (Cdk4) activity and nuclear accumulation of cyclin D1-Cdk4 during G1. J Biol Chem 273: 33279-3386, 1998.

35. Adams JM and Cory S: The Bcl-2 apoptotic switch in cancer development and therapy. Oncogene 26: 1324-1337, 2007.

36. Youle RJ and Strasser A: The Bcl-2 protein family: opposing activities that mediate cell death. Nat Rev Mol Cell Biol 9: 47-59, 2008. 